Progress in Flight Physics 5 (2013) 621-636

DOI: $10.1051 /$ eucass/201305621

(C) Owned by the authors, published by EDP Sciences, 2013

\title{
AERODYNAMIC MODEL DEVELOPMENT AND SIMULATION OF AIRLINER SPIN FOR UPSET RECOVERY
}

\author{
A. $\mathrm{Khrabrov}^{1}$, M. Sidoryuk ${ }^{1}$, and M. Goman ${ }^{2}$ \\ ${ }^{1}$ Central Aerohydrodynamic Institute (TsAGI) \\ Zhukovsky, Russia \\ ${ }^{2}$ De Montfort University \\ Leicester, UK
}

\begin{abstract}
The aerodynamic model of a generic airliner configuration is developed for a wide range of angles of attack, sideslip and angular rate based on experimental data obtained in wind tunnels using static, forced oscillations and rotary balance tests. The developed aerodynamic model is applied for the investigation of the airliner scaled model nonlinear dynamics at high angles of attack with an intensive rotation to identify potential spin modes and spin recovery procedures. The evaluated equilibrium spin parameters and simulated recovery control deflections are in good agreement with the experimental results from free-spin tests obtained in the TsAGI vertical wind tunnel. The work is performed in connection with the European FP7 project SUPRA (Simulation of UPset Recovery in Aviation, www.supra.aero).
\end{abstract}

\section{INTRODUCTION}

A number of flight accidents which have taken place during the last decade are considered to be related to various trigger factors such as severe weather conditions, hardware failures, and pilots' errors leading to aircraft loss-of-control, etc. Analysis of these flight accidents shows that during extreme situations, an aircraft can reach stall conditions where the effects of the flow separation can negatively influence the airliner dynamics. To provide the flight safety in extreme flight situations, it is important to establish a pilot training using available flight simulators to train pilots for upset prevention and recovery control techniques. A simulation of flight upset conditions requires the development of representative aerodynamic models valid in an extended flight envelope that would include extended ranges for the angle of attack, sideslip, and the rotation rate. An 
important part of aerodynamic modeling of airliner upsets is a realistic prediction of the airliner nonlinear dynamics at stall and beyond stall flight conditions, which may lead to an airliner departure and spin entering. An aircraft spin is the most dangerous of critical flight regimes, and its adequate simulation is important for the flight accidents investigation, the design and analysis of control systems for the flight envelope protection, and also for pilots training. The results of this study were obtained in connection with the EU FP7 project SUPRA whose main objective is to develop a representative aerodynamic model for a generic airliner to be used for a pilots' advance training of upset recovery techniques with the help of ground-based flight simulators. This paper presents the outline of the developed aerodynamic model for a generic airliner in a wide range of angles of attack and sideslip accompanied by an intensive rotation about all three axes. The mathematical model contains a number of adjusted parameters that can be tuned to simulate various types of an airliner departure. The aerodynamic model of a generic airliner configuration is developed for a wide range of angles of attack, sideslip, and the angular rate based on experimental data obtained in wind tunnels using static, forced oscillations (in pitch, yaw and roll) and rotary balance tests.

The aerodynamic model's structure is described in section 2 . The important feature of this aerodynamic model is the polynomial approximation of all aerodynamic coefficients vs. the sideslip angle and the rate of rotation (i.e., velocity vector roll rate) where coefficients are functions only of the angle of attack. To expand the approximation of the aerodynamic coefficients to cruise flight speeds, the aerodynamic dependencies in the form of two dimensional lookup data tables are decomposed on two one-dimensional arrays depending separately on the angle of attack and Mach number.

To validate the developed aerodynamic model, the nonlinear aircraft dynamics in the form of equilibrium and agitated spin modes were investigated for the airliner scaled model, which was used in free-spin tests in the TsAGI vertical wind tunnel. In these free-spin tests, time histories for the recorded motion parameter demonstrated highly agitated dynamics; that is why in this paper a special attention was paid to the analysis of periodical spin modes in the form of limit cycles in the dynamic parameters space. The spin dynamics in this paper is investigated computationally using a full system of motion equations and qualitative and bifurcation analysis methods for nonlinear dynamical systems. These nonlinear methods have been widely used in the flight dynamics over the last thirty years [1-8]. Most of previous publications on the bifurcation analysis of the nonlinear dynamics are devoted to a manoeuvrable fighter aircraft, while this study presents results for a generic airliner.

In this paper, a numerical continuation technique was used for the investigation of the airliner equilibrium and periodic solutions of the rigid body nonlinear equations describing the aircraft spatial motion. The local stability analysis using the linearized equations of motion was performed during a continuation 
process in a wide range of control parameters. Stable equilibria and periodic solutions, or closed orbits, correspond to the steady and the agitated spin modes, respectively. To facilitate the computational analysis, the initial values for equilibrium solutions required for the continuation algorithm are computed using an approximate analytical approach. The results of investigation of spin equilibrium and periodic solutions are described in section 3 .

Comments on the comparison of computational predictions of spin parameters and recovery control techniques with free-spin experimental results run in the TsAGI vertical wind tunnel are given in section 4 . The main objective of such comparison was to validate and update the developed aerodynamic model in order to use it for computational and piloted simulation applications.

\section{DESCRIPTION OF THE AERODYNAMIC MODEL}

At low angles of attack with attached flow conditions unsteady aerodynamic effects can be described by damping aerodynamic derivatives, which are normally measured in wind tunnels using a small amplitude forced oscillation technique:

$$
\left.\begin{array}{rl}
C_{N} & =C_{N}(\alpha)+C_{N \varphi}(\alpha) \varphi+C_{N \delta_{e}}(\alpha) \delta_{e} ; \\
C_{m} & =C_{m}(\alpha)+\left(C_{m q}+C_{m \dot{\alpha}}\right) q+C_{m \varphi}(\alpha) \varphi+C_{m \delta_{e}}(\alpha) \delta_{e} ; \\
C_{Y} & =C_{Y \beta}(\alpha) \beta+C_{Y p}(\alpha) p+C_{Y r}(\alpha) r+C_{Y \delta_{a}}(\alpha) \delta_{a}+C_{Y \delta_{r}}(\alpha) \delta_{r} ; \\
C_{l} & =C_{l \beta}(\alpha) \beta+C_{l p}(\alpha) p+C_{l r}(\alpha) r+C_{l \dot{\beta}}(\alpha) \dot{\beta}+C_{l \delta_{a}}(\alpha) \delta_{a}+C_{l \delta_{r}}(\alpha) \delta_{r} ; \\
C_{n} & =C_{n \beta}(\alpha) \beta+C_{n p}(\alpha) p+C_{n r}(\alpha) r+C_{n \dot{\beta}}(\alpha) \dot{\beta}+C_{n \delta_{a}}(\alpha) \delta_{a}+C_{n \delta_{r}}(\alpha) \delta_{r}
\end{array}\right\}
$$

where $p, q$, and $r$ are the roll, the pitch, and the yaw rates, respectively, in the body fixed coordinate frame; $\varphi, \delta_{e}, \delta_{a}$, and $\delta_{r}$ are the deflections of the stabilizer, the elevator, the ailerons, and the rudder, respectively.

The mathematical representation (1) can be used only to simulate the aircraft dynamics at the angles of attack below stall. The analysis of flight accidents with the loss of control situations demonstrates that angles of attack can exceed critical angles of attack with an onset of stall. Airliner departures may lead to the development of an intensive rotation which can significantly affect high angles of attack aerodynamics. This requires appropriate modification of the mathematical model (1) relying on all available experimental data from static tests, small amplitude forced oscillations (in pitch, roll, and yaw) and rotary balance tests. Rotary balance tests correspond to steady aerodynamic conditions when the angular velocity vector is parallel to the air speed vector. However, in free-spin conditions, these two vectors are not necessary parallel, having small attitude misalignments. This kind of aircraft motion is very difficult to simulate 
in a wind tunnel, especially in the dynamic environment. Note that the oscillatory conning data, if they are available, may provide an improved estimation of unsteady aerodynamic effects. The typical way of the aerodynamic modeling of the high angle of attack aerodynamics is to use combined data obtained from rotary balance and forced oscillation tests [5]. Angular rates in the body fixed axes frame can be recalculated to angular velocity vector projections in the velocity axes frame:

$$
\left.\begin{array}{l}
p_{a}=p \cos \alpha \cos \beta+r \sin \alpha \cos \beta+q \sin \beta ; \\
r_{a}=p \sin \alpha-r \cos \alpha ; \\
q_{a}=-p \cos \alpha \sin \beta-r \sin \alpha \sin \beta+\cos \beta
\end{array}\right\}
$$

where $p_{a}$ coincides with the rate of rotation in rotary balance tests and $r_{a}$ and $q_{a}$ components are representing the misalignment between the velocity and the angular velocity vectors. The misalignment components are normally small with respect to rotary balance rate, therefore, the following representation for aerodynamic coefficients may be accepted:

$$
\left.\begin{array}{rl}
C_{X}=C_{X}\left(\alpha, \beta, p_{a}\right)+\Delta C_{X}(\alpha, \varphi)+\Delta C_{X}\left(\alpha, \delta_{e}\right)+\Delta C_{X}\left(\alpha, \delta_{a}\right) & +\Delta C_{X}\left(\alpha, \delta_{r}\right) ; \\
C_{N}=C_{N}\left(\alpha, \beta, p_{a}\right)+\Delta C_{N}(\alpha, \varphi)+\Delta C_{N}\left(\alpha, \delta_{e}\right) ; & \\
C_{Y}=C_{Y}\left(\alpha, \beta, p_{a}\right)+\Delta C_{Y}\left(\alpha, \delta_{a}\right)+\Delta C_{Y}\left(\alpha, \delta_{r}\right) ; \\
C_{m}=C_{m}\left(\alpha, \beta, p_{a}\right)+\Delta C_{m}(\alpha, \varphi)+\Delta C_{m}\left(\alpha, \delta_{e}\right)+C_{m q_{a}} q_{a} ; \\
C_{l}=C_{l}\left(\alpha, \beta, p_{a}\right)+\Delta C_{l}\left(\alpha, \delta_{a}\right)+\Delta C_{l}\left(\alpha, \delta_{r}\right)+C_{l q_{a}} q_{a}+C_{l r_{a}} r_{a} ; \\
C_{n}=C_{n}\left(\alpha, \beta, p_{a}\right)+\Delta C_{n}\left(\alpha, \delta_{a}\right)+\Delta C_{n}\left(\alpha, \delta_{r}\right)+C_{n q_{a}} q_{a}+C_{n r_{a}} r_{a}
\end{array}\right\}
$$

where nonlinear dependences $C_{i}\left(\alpha, \beta, p_{a}\right)(i=X, Y, Z, l, m, n)$ are measured in the rotary balance tests and aerodynamic derivatives with respect to misalignment angular rates are transformed from available forced oscillation tests executed without a steady conical rotation. Further simplification in (3) is made using the following polynomial approximation of the nonlinear dependencies from the rotary balance tests functions:

$$
\begin{aligned}
C_{i}=C_{i 0}(\alpha)+ & C_{i \beta}(\alpha) \beta+C_{i p}(\alpha) p_{a}+C_{i \beta p}(\alpha) \beta p_{a}+C_{i \beta^{2}}(\alpha) \beta^{2}+C_{i p^{2}}(\alpha) p_{a}^{2} \\
& +C_{i \beta^{3}}(\alpha) \beta^{3}+C_{i \beta^{2} p}(\alpha) \beta^{2} p_{a}+C_{i \beta p^{2}}(\alpha) \beta p_{a}^{2}+C_{i p^{3}}(\alpha) p_{a}^{3}
\end{aligned}
$$

where the polynomial coefficients are the functions only of the angle of attack. The aerodynamic derivatives in (3) and (4) can be related to aerodynamic derivatives obtained in small amplitude forced oscillations tests with the account of expressions (2): 


$$
\left.\begin{array}{rl}
C_{l r_{a}}(\alpha) & =C_{l p}^{\mathrm{fo}}(\alpha) \sin \alpha-C_{l r}^{\mathrm{fo}}(\alpha) \cos \alpha ; \\
C_{n r_{a}}(\alpha) & =C_{n p}(\alpha) \sin \alpha-C_{n r}^{\mathrm{fo}}(\alpha) \cos \alpha ; \\
C_{m q_{a}}(\alpha, \beta) & =C_{m q}^{\mathrm{fo}}(\alpha) \cos \beta ; \\
C_{l q_{a}}(\alpha, \beta) & =-\left[C_{l p}^{\mathrm{fo}}(\alpha) \cos \alpha+C_{l r}^{\mathrm{fo}}(\alpha) \sin \alpha\right] \sin \beta ; \\
C_{n q_{a}}(\alpha, \beta) & =-\left[C_{n p}^{\mathrm{fo}}(\alpha) \cos \alpha+C_{n r}^{\mathrm{fo}}(\alpha) \sin \alpha\right] \sin \beta .
\end{array}\right\}
$$

Superscript "fo" in (5) designates the combinations of the rotary and unsteady aerodynamic derivatives measured in small amplitude forced oscillations experiments executed in pitch, roll, and yaw:

$$
\left.\begin{array}{rl}
C_{m q}^{\mathrm{fo}} & =C_{m q}+C_{m \dot{\alpha}} ; \\
C_{l p}^{\mathrm{fo}} & =C_{l p}+C_{l \dot{\beta}} \sin \alpha ; \\
C_{n p}^{\mathrm{fo}} & =C_{n p}+C_{n \dot{\beta}} \sin \alpha ; \\
C_{l r}^{\mathrm{fo}} & =C_{l r}-C_{l \dot{\beta}} \cos \alpha ; \\
C_{n r}^{\mathrm{fo}} & =C_{n r}-C_{n \dot{\beta}} \cos \alpha .
\end{array}\right\}
$$

Finally, representation (3) includes available static data and dynamic aerodynamic derivatives obtained in wind tunnel investigations at a low subsonic flow speed.

\section{SPIN DYNAMICS ANALYSIS}

The developed aerodynamic model of a generic airliner in the form of (3)-(6) was used for computational investigation of the airliner spin dynamics. The continuation and bifurcation analysis methodologies have been used effectively in flight dynamics during the last three decades. The same approach is used in this paper for the analysis of the spin dynamics considering the eighth-order autonomous system of motion equations [5]:

$$
\dot{\mathbf{x}}=\mathbf{F}(\mathbf{x}, \boldsymbol{\delta})
$$

where the state vector $\mathbf{x}=(V, \alpha, \beta, p, q, r, \theta, \phi)^{\prime} \in R^{8}$ and the control vector includes control surface deflections $\boldsymbol{\delta}=\left(\delta_{e}, \delta_{a}, \delta_{r}\right)^{\prime} \in R^{3}$. The equilibrium solutions of this system define a general aircraft motion along a helical trajectory with a vertical axis of rotation. At high angles of attack and a fast rotation, such solutions correspond to equilibrium spin modes. The eighth-order system (7) is considered for the continuation and bifurcation analysis of system equilibria and 


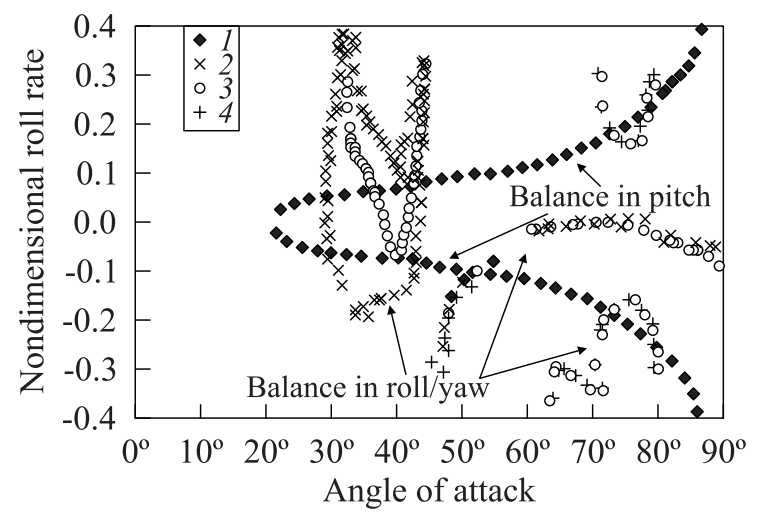

Figure 1 Analysis of approximate solutions for equilibrium spin modes $\left(\delta_{e}=-27^{\circ}\right.$ and $\left.\delta_{r}=27^{\circ}\right): 1-$ balance in pitch; and $2-4-$ balance in roll/yaw $\left(2-\delta_{a}\right.$ $=-27^{\circ} / 15^{\circ} ; 3-0^{\circ} / 0^{\circ}$; and $4-\delta_{a}=15^{\circ} /-27^{\circ}$ )

also periodical solutions or limit cycles. During the continuation of these solutions their local stability analysis using a linearized system of equations is performed. Stable equilibrium points correspond to spins with constant motion parameters, while stable limit cycles correspond to agitated spins with periodical variations of motion parameters.

Control surface deflections $\delta=\left(\delta_{e}, \delta_{a}, \delta_{r}\right)^{\prime} \in R^{3}$ are used as continuation parameters in the continuation process, the throttle position has a much less pronounced effect on state parameters and, therefore, will be kept fixed at its zero position to allow a comparison with free-spin test results. The equilibrium states are defined by the following system of equations represented in a vector form:

$$
\mathbf{F}(\mathbf{x}, \boldsymbol{\delta})=0 .
$$

A continuation procedure needs reasonable initial conditions for state parameters to start continuation. These values were evaluated using approximate equations for an equilibrium spin analysis [5]. The solutions of these approximate spin equations define the balances in all forces and moments. Figure 1 shows isolines representing the equilibrium in the longitudinal and the lateral/directional aircraft motion in the plane of angle of attack and dimensionless velocity roll rate. The crossing points of these isolines represent a full set of possible equilibrium flight regimes, with some of them being stable and some unstable. Not all of approximate solutions can result in spin solutions for the full set of equations but they are useful as initial values for a continuation procedure applied to the full system (7) for obtaining a total picture of spin solutions.

In all presented results, the rudder deflection $\delta=\delta_{r}$ is used as a continuation parameter, while remaining control parameters $\delta_{e}$ and $\delta_{a}$ are kept fixed. The 
continuation of equilibrium solutions was performed for different combinations of these parameters.

The continuation of limit cycles or periodical solutions is performed by solving the following parameter dependent boundary-value problem

$$
\mathbf{x}(T, \delta)-\mathbf{x}(0, \delta)=0
$$

where the oscillations period $T$ is one of the unknown variables. To solve the vector equation (8) correctly, the initial value of one of state variables from $x$ should be fixed within the limit circle variation, for example, initial sideslip angle is set to be zero: $\beta(0)=0$.

Continuation results for equilibrium and periodic solutions of system (7) against the rudder deflection are shown in Figs. 2-4. Unstable equilibrium brunches of solutions are marked in grey and stable solutions are marked in black. Stable limit circles are shown via plotting their minimal and maximal values at different rudder deflections $\delta_{r}$. Unstable branches for limit cycles are not displayed to avoid overloading the presentation of obtained results. As can be seen, a picture of spin solutions and different types of bifurcations is rather rich. One of the basic goals of the current study is to show a rich set of dangerous stable spin modes in a wide range of control deflections rather than detailed bifurcation analysis. Below several bifurcations are indicated. Thus, a number of Hopf bifurcation points (where a complex conjugate pair of eigenvalues of the stationary solution intersects the imaginary axis) can be seen in Figs. 2-4. In Fig. 2, in a curve of steady spins at approximately $\delta_{r}=5^{\circ}$, a subcritical Hopf bifurcation point is available. In Figs. 3 and 4 , a supercritical and subcritical Hopf bifurcation points are located approximately at $\delta_{r}=12^{\circ}$ and $18^{\circ}$, respectively. These bifurcation points give initial parameters for the calculation of oscillatory spin modes via the continuation procedure for solutions of Eq. (8). Examples of computed closed orbits are shown in Figs. 5 and 6 in the form of phase portrait projections and also in the form of time histories.

Periodic solutions are also subjected to different bifurcations that are more complicated than bifurcations of steady solutions. For example, in Fig. 2, at $\delta_{r}=-3.6^{\circ}$, a stable limit circle of period $T=0.86 \mathrm{~s}$ loses stability via periodic solution multiplier crossing through -1 . At the same time, a stable doubling period $T=1.72 \mathrm{~s}$ solution occurs. In a narrow range of elevator deflections (less than $1^{\circ}$ ), a sequence of period doubling bifurcations leading to onset of chaos is obtained. Chaotic trajectories at $\delta_{e}=-27^{\circ}, \delta_{a r}=-27^{\circ}$, and $\delta_{a l}=15^{\circ}$ exist in the range of rudder deflections $-3^{\circ}<\delta_{r}<3^{\circ}$. Figure 7 presents a sequence of phase portrait projections and time histories demonstrating the bifurcations of limit circles through period doubling to chaotic attractor at $\delta_{e}$ $=-27^{\circ}, \delta_{a r}=-27^{\circ}$, and $\delta_{a l}=15^{\circ}$. Note that the period of aircraft rotation (yaw angle $\psi$ is the coordinate which is integrated separately from the 8th-order 

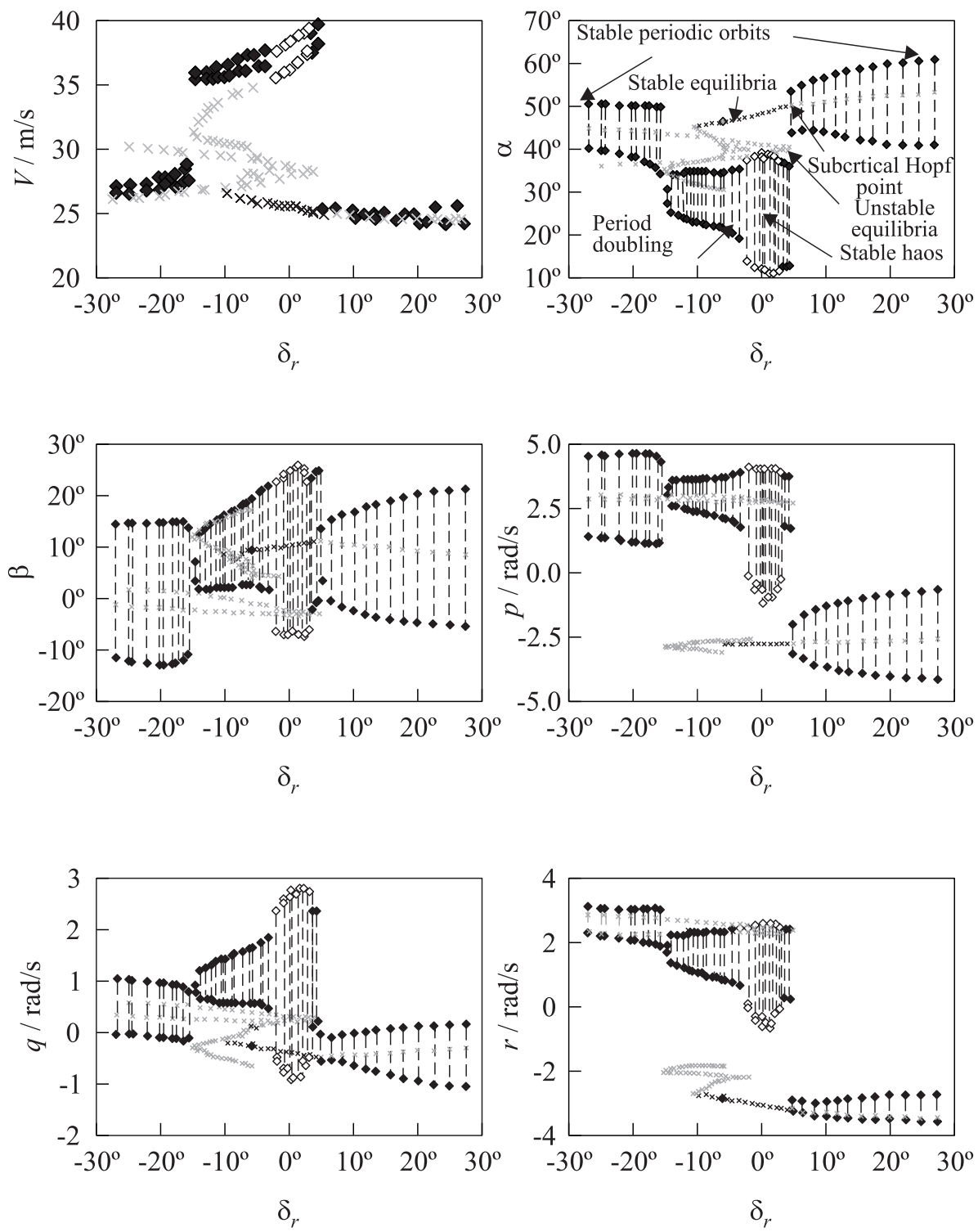

Figure 2 Equilibrium, stable periodic, and chaotic solutions for different rudder deflections at $\delta_{e}=-27^{\circ}, \delta_{a r}=-27$, and $\delta_{a l}=15^{\circ}$ 

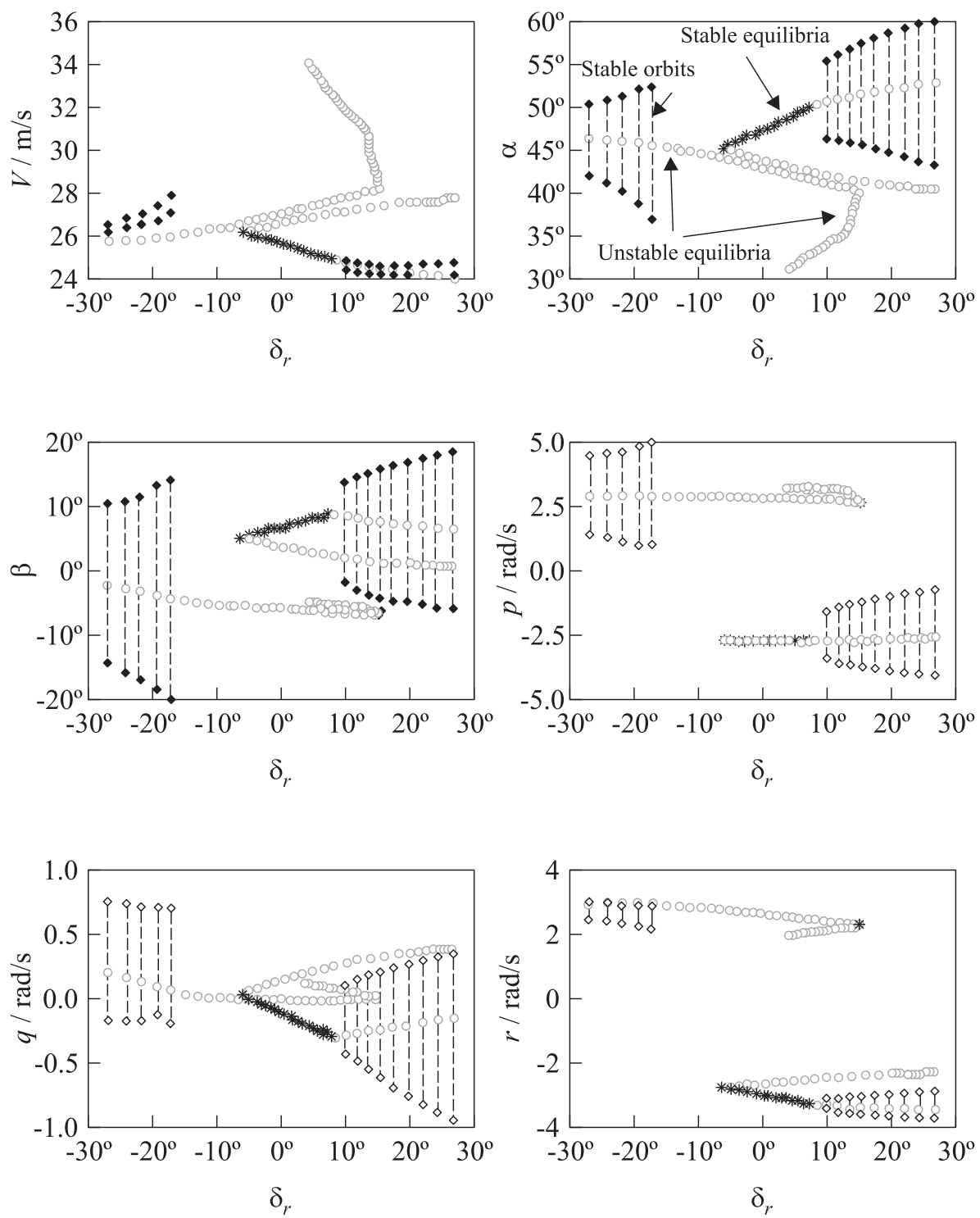

Figure 3 Equilibrium, stable periodic solutions for different rudder deflections at $\delta_{e}=-27^{\circ}$ and $\delta_{a}=0^{\circ}$ 

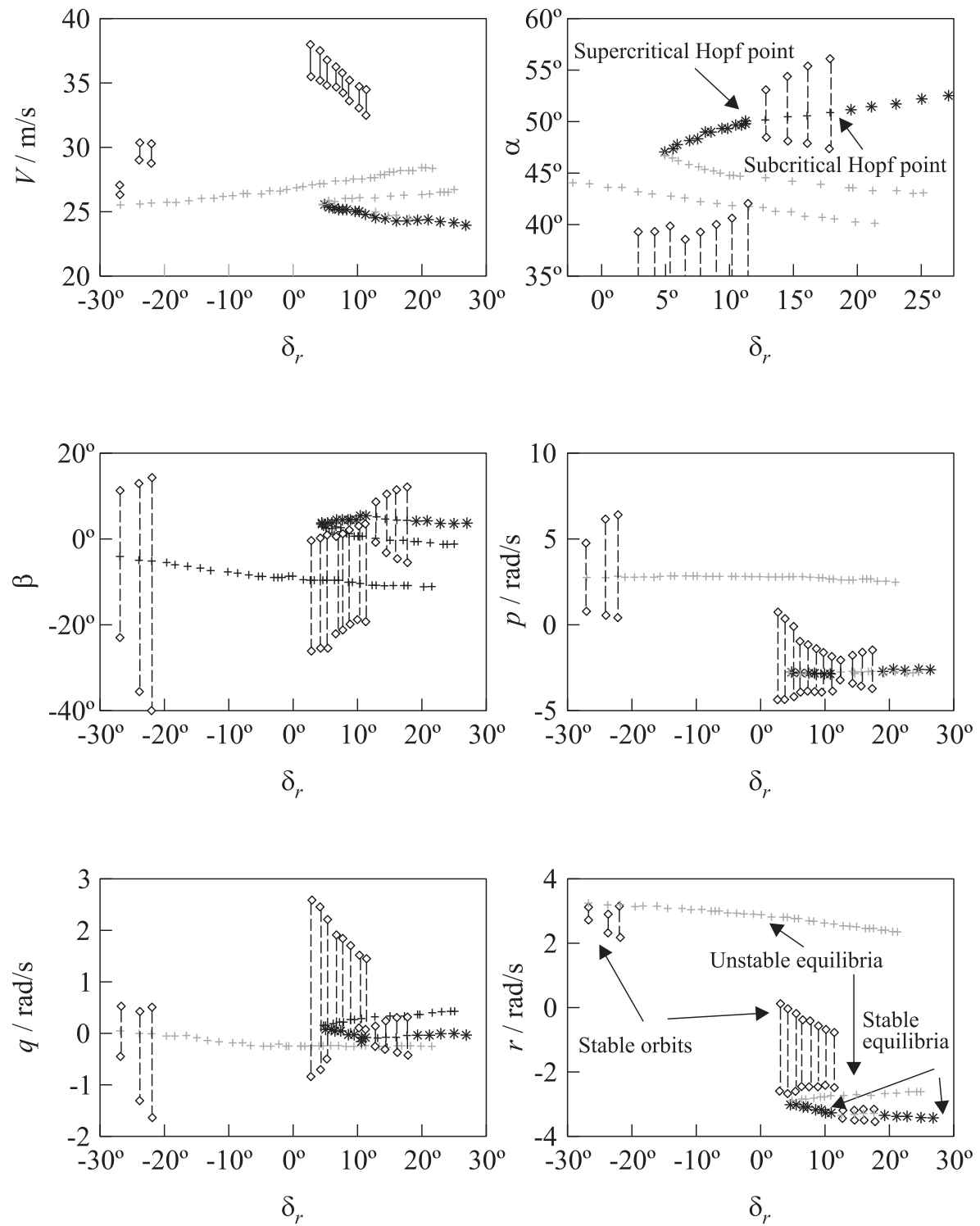

Figure 4 Equilibrium, stable periodic solutions for different rudder deflections at $\delta_{a r}=15^{\circ}, \delta_{a l}=-27^{\circ}$ 

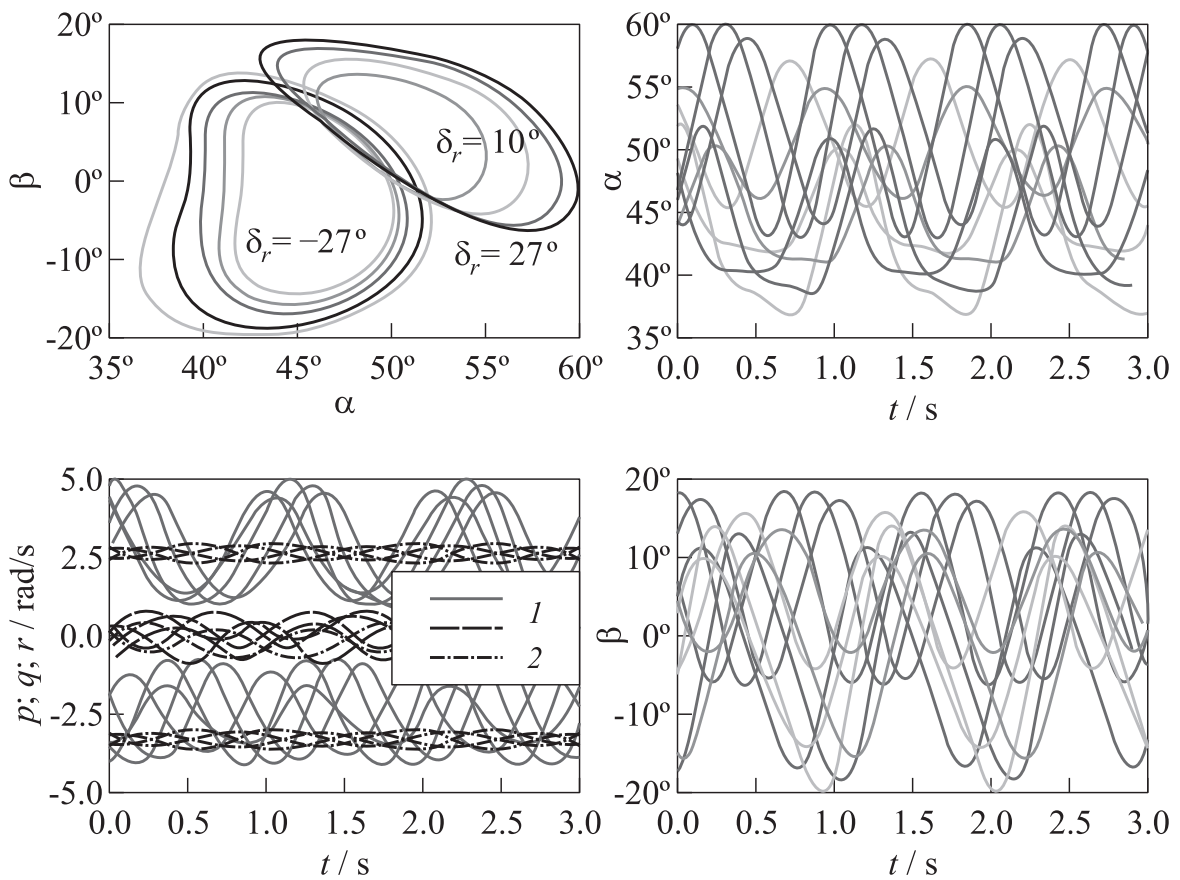

Figure 5 Periodic solutions describing oscillatory spin for rudder deflection: $\delta_{a}=0$

system (7)) is not doubled during a period double bifurcation and chaos onset. Notice also that both stable spin modes with periodic or chaotic changing of parameters are equally dangerous from a practical point of view.

Some of the presented limit circles originate at bifurcation points that lie outside physical control deflection limits. Analysis of the presented results shows that different stable spin modes coexist at the same control deflections. So, stable spin modes are available both at 45-60 degree and 27-40 degree angles of attack. At angles of attack about $70^{\circ}-80^{\circ}$, the stationary spin solutions are unstable (some of them are shown in Fig. 1 representing an approximate spin analysis). Here, no stable spin solutions were found.

\section{SPIN RECOVERY SIMULATION}

Computational results of the spin dynamics simulation are used for a comparison with experimental time histories of the dynamically scaled model obtained in free-spin tests in the TsAGI vertical wind tunnel. These results were kindly provided by M. Golovkin, A. Efremov, and V. Gorbunov. Such comparison of 

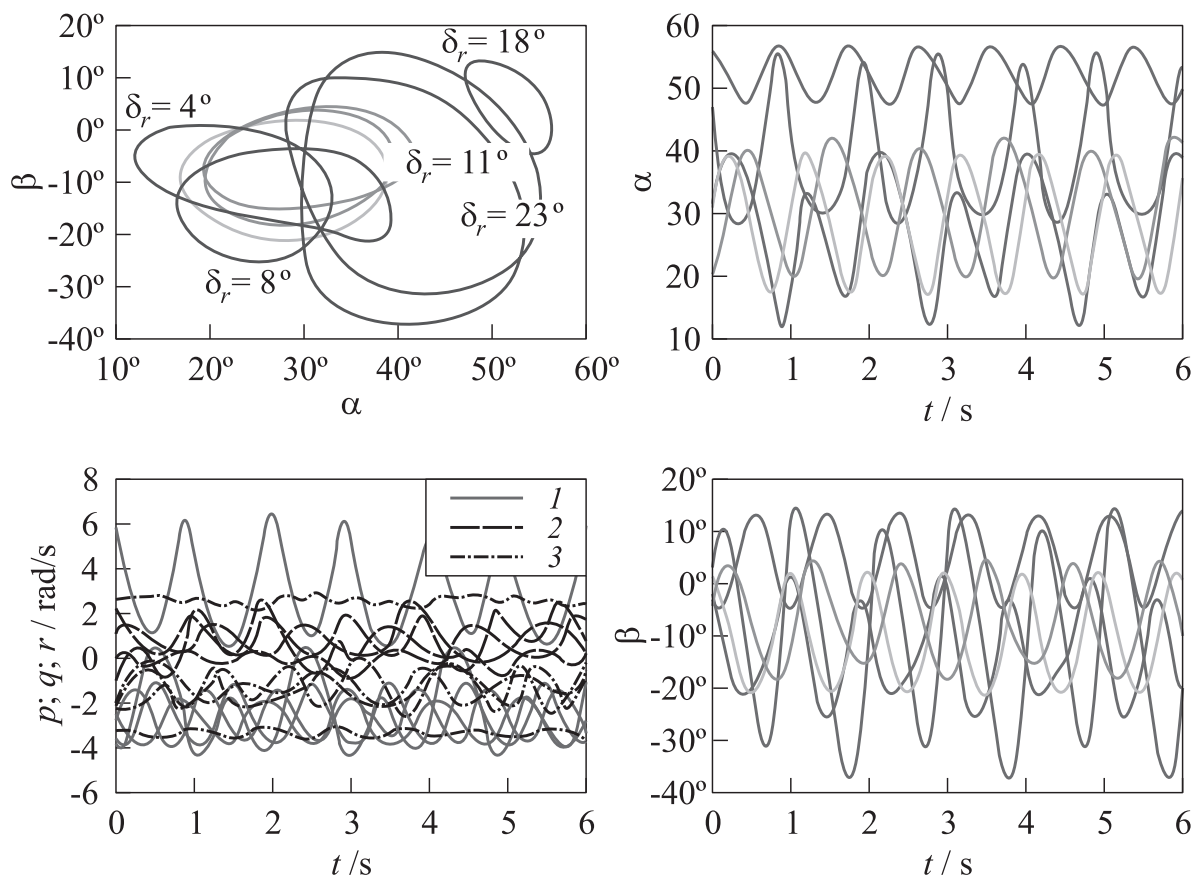

Figure 6 Periodic solutions describing oscillatory spin for rudder deflection: $\delta_{a r}$ $=15^{\circ}, \delta_{a l}=-27^{\circ}$

computational and experimental time histories can be extremely valuable for the validation of the developed aerodynamic model.

The dynamically scaled model in the wind tunnel was equipped with sensors and actuators. A radio communication channel is used for the data acquisition and also transmitting recovery control program to the free-spin model. $\mathrm{Nu}-$ merical simulations of the spin motion and the recovery from the spin were conducted with the same time control sequences which were implemented in the experiment. Comparisons of computationally predicted and experimentally obtained dynamics time histories were performed for different initial settings and control recovery deflections. The compared spin dynamics characteristics included the average values and ranges of variation for the angle of attack, the sideslip angle, and the spin rate. An example of such numerical simulation of a developed spin and an implementation of experimental recovery control program is presented in Fig. 8. One can see that numerically simulated trajectories are quite close qualitatively and quantitatively to the experimental free-spin time histories (Fig. 8a). The spin rate is very similar and the recovery from the developed spin occurs practically at the same 

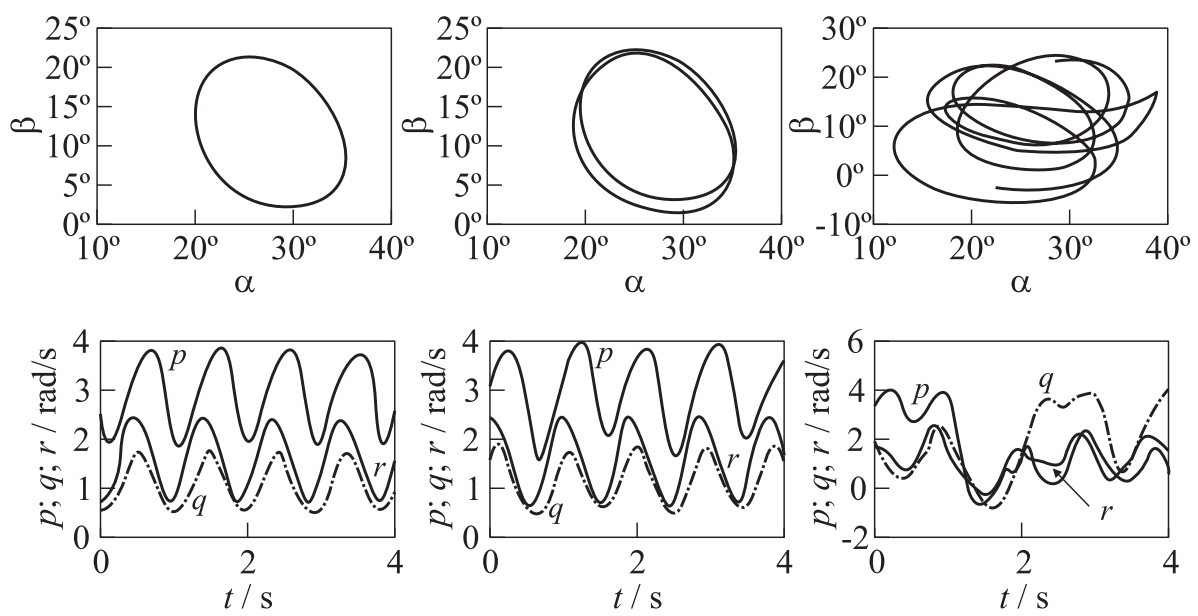

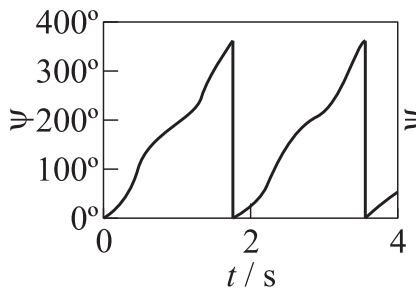

(a)

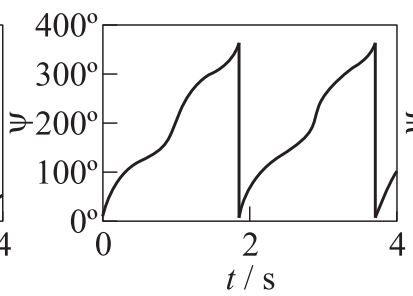

(b)

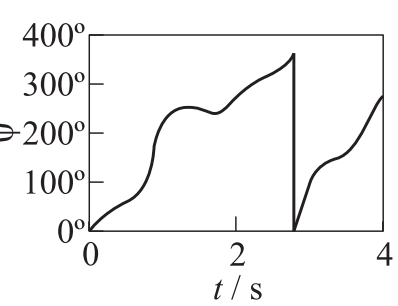

(c)

Figure 7 Period doubling bifurcations leading to onset of chaotic attractor in spin dynamics at $\delta_{e}=-27^{\circ}, \delta_{a r}=-27^{\circ}$, and $\delta_{a l}=15^{\circ}:(a) \delta_{r}=-4.1^{\circ} ;(b)-3.4^{\circ} ;$ and (c) $\delta_{r}=-0.7^{\circ}$

time. Average angles of attack in the experimental and the numerical simulations in this example are $50^{\circ}$ and $47^{\circ}$, respectively. The velocity in spin $V=26 \mathrm{~m} / \mathrm{s}$ and the flow speed in the vertical wind tunnel were very close: $V=24 \mathrm{~m} / \mathrm{s}$.

A period of one turn is approximately $T=1.62 \mathrm{~s}$, both in the experiment and in the numerical simulations. One can hardly expect higher closeness in state variables, because totally settled oscillatory spin requires about $20-30 \mathrm{~s}$, it means that there is very high sensitivity to initial conditions taken in numerical simulation. The free-spin experiments were performed, mainly, for different extreme deflections of all control surfaces. The typical control strategy applied in free-spin experiments for the spin recovery is as follows: ailerons and the rudder are simultaneously deflected in the opposite extreme positions and, after the airliner model makes one half turn, the elevator is deflected to the opposite extreme position to produce a recovery action. This control strategy was effec- 

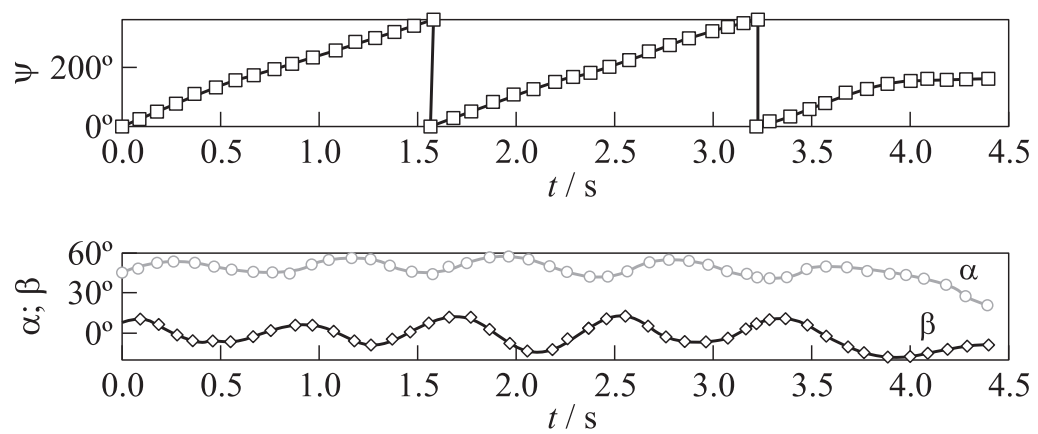

(a)
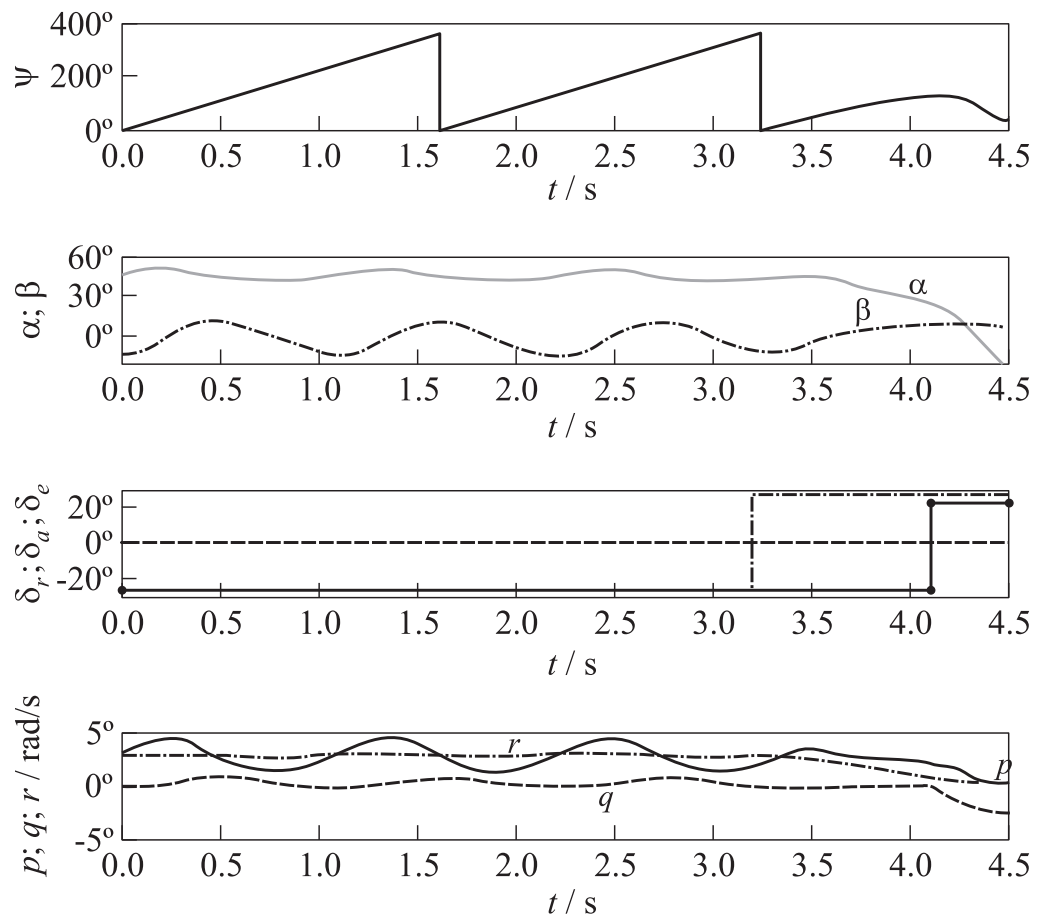

(b)

Figure 8 Comparison of experimental $(a)$ and computational $(b)$ time histories for motion parameters in oscillatory spin and simulation of spin recovery control 
tive in most cases for the spin termination and the recovery from high angles of attack to normal conditions.

\section{CONCLUDING REMARKS}

The presented computational investigation of an airliner flight dynamics with the developed aerodynamic model for an extended flight envelope has demonstrated very good qualitative and quantitative agreement with the results of free- spin experiments in the vertical wind tunnel. This can be considered as validation of the developed aerodynamic model, because it was created using experimental data from different wind tunnel tests using forced oscillation and rotary balance methods. This is an important step in the validation of the SUPRA aerodynamic model, which will be used for piloted simulation in TsAGI (Russia), and NLR and TNO (Netherlands) for upset recovery training. The representative aerodynamic model will help to avoid the effect of negative pilot training. However, there is still room for improvement in quantitative matching of computationally predicted and experimental time histories. For example, the damping aerodynamic derivatives used in the aerodynamic model were obtained in experimental tests with zero mean value of the conical angular rate. To investigate the influence of an intensive airliner rotation on aerodynamic damping derivatives, additional wind tunnel experiments with oscillatory conning motion for an airliner model should be executed.

\section{ACKNOWLEDGMENTS}

This work was performed in connection with the authors involvement in the European FP7 project SUPRA (www.supra.aero). The authors also acknowledge the valuable help and advice from M. Golovkin, A. Efremov, and V. Gorbunov, who also provided the free-spin time histories obtained in the TsAGI vertical wind tunnel which were used in this study. This work was partly supported by the Ministry of Education and Science of the Russian Federation, projects Nos. 14.U01.21.8377 and 14.U01.21.8759, and Russian Foundation for Basic Research, project No. 12-08-00679-a.

\section{REFERENCES}

1. Carroll, J. V., and R. K. Mehra. 1982. Bifurcation analysis of nonlinear aircraft dynamics. J. Guidance Control Dyn. 5(5):529-36. 
2. Zagaynov, G.I., and M.G. Goman. 1984. Bifurcation analysis of critical aircraft flight regimes. Toulouse, France. ICAS Paper No. 84-4.2.1.

3. Guicheteau, P. 1990. Bifurcation theory in flight dynamics - an application to a real combat aircraft. Stockholm. ICAS Paper No. 90-5.10.4.

4. Goman, M. G., G. I. Zagaynov, and A.V. Khramtsovsky. 1997. Application of bifurcation methods to nonlinear flight dynamics problems. Prog. Aerospace Sci. 33(59):539-86.

5. Bushgens, G. S., ed. 1998. Aerodynamics, stability and controllability of supersonic aircraft. Moscow: Nauka, Fizmatlit. 816 p. [In Russian.]

6. Guicheteau P. 1998. Bigurcation theory: A tool for nonlinear flight dynamics. Phil. Trans. R. Soc. L. Ser. A 356:2181-201.

7. Goman, M. G., and A. V. Khramtsovsky. 1998. Application of continuation and bifurcation methods to the design of control systems. Phil. Trans. R. Soc. L. Ser. AMath. Phys. Eng. Sci. 356(1745)2277-95.

8. Raghavendra, P. K., P. Tuhin Sahai, A. Kumar, M. Chauhan, and N. Ananthkrishnan. 2005. Aircraft spin recovery, with and without thrust vectoring using nonlinear dynamic inversion. J. Aircraft 42(6). 